\title{
Portfolio Optimization Model Based on CVaR Programming and Limits of MAD \\ Jinsheng $\mathrm{CaO}^{1, \mathrm{a}}$
}

${ }^{1}$ Department of Investment, Zhongnan University of Economics and Law, Wuhan 430070, China

a893287703@qq.com

Keywords: Portfolio; CVaR Programming; MAD

\begin{abstract}
Portfolio problem is one of the hotspots of the current financial theory anthe main research at the desired rate of return is determined to find the premise of asset alloc ation investment program, or in the case of identified risks to maximize profits. Early research portfolio focused on portfolio income measure, then the transition to measure portfolio risk. This paper presents a CVaR portfolio model based on a combination of capital gains rate not assume a normal distribution, with the MAD model as a constraint, realized volatility measure limit, spend a convex utility function as a constraint, indicating risk asset transaction costs. Experimental results show that the model meets the requirements of the actual investment, in line with the actual investment law, and MV CVaR model and the original model and compared with the volatility of the value at risk minimization advantage.
\end{abstract}

\section{Introduction}

Since the 1990s, economic globalization, financial integration is increasingly apparent. With the increased mobility of transnational capital, the original capital is limited within a reasonable distribution worldwide. At the same time, due to the rapid development of modern financial theory and financial engineering techniques, variety of transactions in financial markets, trading volume and transaction speed has been further improved, and to promote options, futures, insurance and other financial derivatives and swaps transactions widely in the financial markets. What followed was a virtual economy countries run the risk of a significant increase. Stochastic risk and fuzzy risk and other new financial risk has gradually replaced the traditional market risk, as the main risk facing the global financial community. Because the risk of an objective system exists in the financial markets, it is a reasonable use of financial risk management has become the key to sustained and healthy economic development of countries [1].

In order to deal with the growing financial market risks, apart from the national level and the level of financial regulators risk constraints and limitations beyond, but also make full use of all economic entities portfolio method to avoid and distract potential risks. As a result, portfolio theory emerged. Accurate and efficient portfolio model is an effective tool to guide financial institutions and corporate investment or distribution of assets, it can help to some extent, the economic entity to avoid potential financial losses.

Currently, the portfolio theory has become one of the four pillars of modern financial theory. Its use of hedging, dispersing and adjust the capital limits and other methods to control risk in the process played a fundamental role in the field of theoretical research and practical applications in achieved fruitful results. Metrics for analyzing earnings volatility model portfolio mainly mean variance (Mean- variance, MV) model [2]. There are values (Value at risk, VaR) model in insurance against the risk of loss probability and the value of the portfolio risk models. However, these methods are only from one or both sides of the description and measure risk, meaning the risk of failure to proceed to complete included to measure risk. Quantitative investment, especially in model portfolios, risk considerations must include both the definition of risk, so integrated risk model portfolio volatility and risk value is of great importance. 


\section{The Concept of CVaR Model}

The fundamental purpose of the portfolio is to guide investors to diversify the use of reasonable methods to make to balance the benefits and risks. Markowitz established mean - variance MV model describes the combination of risk and expected return relationship. Fluctuation variance of the model includes both the losses, but also includes gains, but volatility should not be included in the loss of revenue, followed by volatility can only describe some of the characteristics of risk. Konno and Yamazaki average deviation proposed model, the optimization problem of discrete case is converted to a linear optimization problem by measuring the size of volatility to measure risk, but you can not calculate the mean of the portfolio losses. JP Morgan proposed a method to measure the downside risk assets VaR (Value at Risk), may, within the overall framework consistent with only one easy-to-understand figures to measure the market risk of the portfolio consisting of many complex tool, but can not meet the agreed risk measure theory. Rockefeller and Joseph Yule CVaR model is proposed, when asset returns follow a normal distribution, can be well described by the efficient frontier portfolio, but underestimate the actual risk of major losses. In addition, although the model is consistent risk measure theory meet and comply with convexity axiom, but can not control the volatility of portfolio risk [3].

This paper presents an improved model of CVaR, the volatility of the MAD model as CVaR constraints added to the model, so that the model can be controlled CVaR volatility, the transaction fee by the convex utility function and write the constraint conditions solving through historical simulation CVaR model, thus avoiding significant losses due to data distribution tail is too large may cause.

CVaR model as improved model VaR model, which is subject to any conditions are met under the assumption that four axioms coherent risk measure in yield, consistency risk measurement methods. The main advantage of CVaR models have: (1) CVaR model is not a measure of risk into a single probability point value, which is the tail of the loss expectations. Through comprehensive consideration of all information is greater than the $\mathrm{VaR}$ tail to avoid the occurrence of extreme losses ignore the possibility. (2) from the CVaR model definition, the value of CVaR model depends VaR values, which were divided in two directions from the site, and to limit the risk of the average of the tail. (3) CVaR model meets coherent risk measure, it is a convex optimization problem, which there is a global optimal solution. (4) CVaR model uses money as a risk measurement unit, easy to understand and use [4].

\section{Portfolio Optimization Basis}

The Measurement of Portfolio Yield. In the investment process, investors will generally choose to measure return on assets, return on invested assets, the following two reasons. First, the yield is an investment opportunity for the overall summary. Second, the investment rate of return has good statistical properties, it is easier to handle than the price series. According time characteristics, yield investment assets into simple yield and yield two consecutive. Measure a group of assets yield index mainly median, mode, and other methods of mathematical expectation.

The median is less susceptible to the extreme impact of the advantage, the advantage is that the larger the probability of the mode in which they appear in the return series, the two drawbacks are not fully measure the entire income series. So, whether it is still in the practical application of theoretical calculations, mathematical expectation is often the preferred method [5].

The Measurement of the Portfolio Transaction Costs. In the actual transaction process, such as the presence of transaction costs, dividends and tax transactions such friction. These transactions friction in the case of a small volume of transactions can be neglected, but in the case of a large volume of the transaction process will play a decisive impact. Transaction costs which are investor turnover ratios of the respective fees paid to the exchange, because a larger proportion of the trade friction and get the attention of investors. Arnoot and WagneR study ignores the possibility that the transaction costs arising from ineffective portfolio strategy. Edwin in his monograph also 
highlighted the impact of transaction costs on the risk of investment. Calculation method depends on transaction costs by treating each exchange or trading transaction costs attitude. Patel and Subrahmanyam studied the issue for a fixed rate investment case on the assumption that transaction costs. Yoshimoto think the investment process is variable transaction costs. Under the influence of this idea, Mulvey proposed transaction by convex function to approximate the cost function [36]. Konno Lee proposed transaction concave function to approximate the cost function.

The Measurement of Portfolio Risk. From the perspective of the definition of risk, the risk of this article will be divided into random risk and fuzzy risk. Where the stochastic risk in the investment process is the uncertainty of the corresponding risk in the portfolio model includes the volatility of asset returns. Fuzzy risk corresponds to the risk of loss of value of the uncertainty in the investment process, the portfolio model includes loss probability and risk value.

MAD Measurement. Volatility is a measure of an important indicator of the extent of capital gains rate fluctuations. It is also the investment risk metrics random direction. MV Markowitz proposed model is a typical representative of this idea. MV model but there are some deficiencies in the yield and fat tail risk of the portfolio can not fully describe the emergence of distribution. Sharpe affected by the proposed use instead of the standard deviation of the mean difference mitigate outliers portfolio analysis. MAD model portfolio model is based on the above ideas established by the measure of its future capital gains rate expected rate of return to measure the degree of deviation from the analysis of investment risk. The average difference from the definition:

$$
\delta_{x}=E\left|\sum_{i=1}^{n} x_{i} r_{i}-E\left(\sum_{i=1}^{n} x_{i} r\right)\right|=E|r-\mu|
$$

Where, $\delta_{x}$ is the average number of $\mathrm{P}, \mu$ is the yield ratio average number.

VaR Measurement. Due to the volatility of the portfolio based on the traditional model can not measure the loss of value of the investment, Goldimann proposed VaR model. Its meaning is "a certain probability (confidence level) under a portfolio of financial assets or in a certain holding period expected value of the maximum possible loss." Which indicates a certain probability $\mathrm{P}$ portfolio in a certain time period confidence, the probability of losses in excess of the maximum expected value of $\mathrm{VaR}$ for 1 . Because the model is added to the probability of this constraint entry, so that the model makes up for MV and MAD and other models from the direction of the ambiguity of the risk of defects [5].

According to the definition of $\mathrm{VaR}$ models, $\mathrm{VaR}$ model expressions loss values are:

$$
\operatorname{prob}(\nabla p>V a R)=1-\beta
$$

Where, $\beta$ is the confidential number of VaR model, $\nabla p$ is the loss in t period.

CVaR Measurement. 1999, ArtzneR proposed coherent risk measure. The standard measure by monotony, translation invariance, positive homogeneous and subadditive measure and compare the prevailing model portfolios. By this indicator, Artzner, Roekafella and Uryase others within the framework of consistent risk measure VaR model studies. The research concluded that the VaR model does not satisfy subadditivity, so it does not meet the coherent risk measure. In 2004, Huang Xiangyang, Measured by research-based portfolio optimization model Conditional Value at Risk. On this basis, RockafellaR and Uryasev CVaR model proposes an improved model VaR model in 2002. Meaning CVaR model is "in the case of a certain degree of confidence and holding period, the loss of the portfolio VaR exceeds a given value, the average value of the portfolio losses, reflecting the loss of part of the average level of excess." 2001 Liushan deposit, Shouyang Wang of China's financial markets combined with game theory methods proposed investment analysis. By definition, the expression is:

$$
\operatorname{CVaR}_{\beta}=E|f(x, y)| f(x, y)>\operatorname{VaR}_{\beta}
$$

Where, $f(x, y)$ is Portfolio expected loss.

\section{Portfolio Optimization Model Based on CVaR Programming and Limits of MAD}

Currently, the risk of uncertainty and risk the loss of two ways to measure the uncertainty in the 
model portfolio investment has matured and is showing a systematic development trend. Risk Metrics theory at the same time as the portfolio model development. But the two models have advantages and disadvantages, the two models for effective integration has important application value.

MAD model is a combination of model-based investment rate of return of investment volatility risk. With respect to the MV model has a simple structure, easy to calculate the characteristics and the average absolute deviation can effectively reduce the impact of outliers or extreme risk. MAD model but can only measure the volatility risk, the risk of loss can not be given a value in monetary units. Thus, the model can not fully measure the risk and give investment asset allocation plan.

CVaR model is a model investment portfolio losses based on the value of the investment portfolio risk. With respect to the VaR models, subject to the consistency of the model risk measure, convex programming model to meet the requirements, and the average of these two directions, respectively, from the sub-site restrictions and tail risk. But CVaR model can only measure the value of risk from the risk of loss in this direction, for limiting the volatility of the portfolio rate of return can not do anything.

Proposed a combined model single investment CVaR based on a combination of capital gains rate not assume a normal distribution, with the MAD model as a constraint, realized volatility measure limit, spend a convex utility function as a constraint, expressed risk assets transaction costs. Experimental results show that the model meets the requirements of the actual investment, in line with the actual investment law, and MV CVaR model and the original model and compared with the volatility of the value at risk minimization advantage.

\section{Conclusion}

This paper presents a model investment portfolio based on a CVaR using MAD model as portfolio volatility constraints, using a convex utility function transaction rates, making CVaR model has more practical. In addition, the calculation of $\mathrm{CVaR}$ model uses historical simulation, thus avoiding the assumption of normal distribution of data. Experimental results show that the model can help improve investment efficiency, reduce risk and has good prospects.

\section{References}

[1] Marko Wiz.: The Journal of Finance, Vo1. 4 (1952) No. 26, p.77-79.

[2] Mclntire KL, Asher MA, Burton DC,Liu W, Wang Fucheng: Calculate and Application, Vo1. 4 (2010) No. 5, p. 411-419.

[3] Fok CL, Li Changhong, etc.: Systems Engineering Theory and Practice, Vo1. 34 (2009) No. 11, p. 1608-1618.

[4] Sun Qibo: Journal of Anhui University of Technology, Vo1. 23 (2010) No. 26, p. 786-793.

[5] Liu Jie, Li Shan: Value Engineering, Vo1. 31 (2007) No.27, p. 260-264. 